\section{Secondary prevention of hidradenitis suppurativa}

\author{
Hjalmar Kurzen, ${ }^{1,2}$ Marina Kurzen ${ }^{1}$ \\ ${ }^{1}$ Freising Skin and Laser Center, \\ Freising; ${ }^{2}$ Department of Dermatology, \\ Venereology and Allergy, University \\ Medical Center Mannheim, Ruprecht- \\ Karls University of Heidelberg, \\ Mannheim, Germany
}

\begin{abstract}
Hidradenitis suppurativa (HS) is a multifactorial disease with many facets of uncertain importance for optimal treatment and prevention. In order to explore options for secondary prevention in HS, we randomly and retrospectively selected 40 patients with HS that were analyzed on the basis of supposed trigger factors and proposed prevention measures. $67 \%$ of our HS patients were current smokers. They had started smoking on average 8 years prior to abscess formation. $35 \%$ complained of digestive problems and had tried different sorts of diet. We identified 2 cases of gluten-sensitive enteropathy, in which HS improved after introduction of gluten-free diet. In 7 further patients, introduction of low dairy/low carbohydrate diet considerably improved HS. $77.5 \%$ had never used any skin care in the intertriginous areas. Implementing secondary prevention by reducing irritation, avoiding shaving, improving skin care, performing laser epilation or applying fusidic acid/betamethasone cream led to an improvement in $62.5 \%$ of patients. We suggest a structured approach in daily practice in order to identify individual trigger factors. The crucial point for secondary prevention is the improvement of patient education.
\end{abstract}

\section{Introduction}

A better understanding of the immunological pathologies and the approval of the first drug licensed for hidradenitis suppurativa (HS), adalimumab (ADA) has drawn the focus of the dermatological community to the treatment of advanced cases of HS for which ADA has been examined. ${ }^{1}$ However, in our daily practice, we still have to start the treatment at the beginning. HS is a puzzling disease, defined as chronic debilitating inflammatory disorder with main involvement of hair follicle-bearing areas of the skin folds. ${ }^{2}$ Its pathogenesis remains unclear and its name remains disputed despite considerable advances. ${ }^{3,4}$ Around
$40 \%$ of HS patients suffer from a familial form of HS that may be different from sporadic forms of HS. ${ }^{2}$

Prevention is considered to be the ultimate goal in medical practice. ${ }^{5}$ Primary prevention aims to prevent disease or injury before it ever occurs. ${ }^{6}$ In the case of HS this remains not possible unless the pathogenesis will be fully understood and prospective disease markers will be made available. Secondary prevention aims to reduce the impact of a disease or injury that has already occurred. It has been proposed that the primary lesion of HS may be a spongiform infundibulofolliculitis and perifolliculitis in more than $95 \%$ of cases. $^{3}$ Secondary prevention implies prevention of the infundibulofolliculitis from becoming a deep folliculitis, nodule or fistula. It also means prevention of a patient with Hurley stage I from progressing to Hurley stage II or III. Tertiary prevention aims to soften the impact of an ongoing illness or injury that has lasting effects. In HS, this could be translated to reducing the impact of inflammation, fistulas and scars on daily life. ${ }^{6}$

Worldwide, HS patients today still suffer from a considerable diagnostic delay of 8-12 years between first symptoms and accurate diagnosis. ${ }^{4}$ As a direct consequence, no adequate information on the nature of the disease, possible preventive strategies or adequate treatments are given to the patients. For example, incision of acute abscesses results in almost all cases in recurrences, scar and fistula formation, while deroofing or wide excisions have very low recurrence rates. ${ }^{2,4}$ The term or concept of prevention is not mentioned in the current European or US HS guidelines. ${ }^{2,7}$ However, based on current available data, prevention strategies can already today be communicated to HS patients in order to reduce disease burden and stop the progression to more severe HS stages. Recently, we introduced a concept of secondary prevention of HS. ${ }^{8}$ In accordance with current pathogenic concepts ${ }^{3}$ and welldefined trigger factors like obesity, smoking, sweating or mechanical irritation ${ }^{3,4}$ we sought to determine which information was the most important one to give to the patient in order to influence the further course of the disease. Based on a first concept of secondary prevention we developed a catalogue of questions, that was used in daily practice by different doctors. The aim was to improve anamnestic accuracy and to identify individual trigger factors in order to be able to propose a modification of the identified factors instead of giving the same recommendations to every patient.
Correspondence: Hjalmar Kurzen, Freising Skin and Laser Center, Kesselschmiedstr. 2, 85354 Freising, Germany.

Tel. +49.8161 .9353090 - Fax: +49.8161.9353099.

E-mail: hjalmar.kurzen@nexgo.de -

kurzen@prof-kurzen.de

Key words: Acne inversa; Preventive measure; Therapy; Trigger factor; Friction; Smoking.

Contributions: HK designed the study, performed analyses and wrote the manuscript; MK co-designed the study, discussed all results and proofread the manuscript.

Conflict of interest: the authors declare no potential conflict of interest.

Funding: none

Received for publication: 21 July 2019 Revision received: 25 August 2019.

Accepted for publication: 25 September 2019.

This work is licensed under a Creative Commons Attribution-NonCommercial 4.0 International License (CC BY-NC 4.0).

${ }^{\circ}$ Copyright: the Author(s), 2019

Licensee PAGEPress, Italy

Dermatology Reports 2019; 11:8243

doi:10.4081/dr.2019.8243

\section{Materials and Methods}

From $02 / 2016$ to $12 / 2018$ all HS patients that came to our clinic were routinely screened for trigger factors and comorbidities. We retrospectively analyzed a subset of patients that had returned at least for a second visit. In 40 patients age, gender, smoking habits, body weight and height, duration of disease, family history of HS and Hurley stage was determined. Dietary restrictions or influence of certain food compounds on HS severity were assessed. We further asked for digestive problems, sweating, shaving habits and skin care procedures in the affected areas (our catalogue of questions is shown in Table 1). Finally, on the second visit, we wanted to know whether proposed preventive strategies had turned out to be helpful in the course of the disease. All patients had been informed and consented the anonymous use of their data. Statistical analysis was performed using a t-test available on www.graphpad.com.

\section{Results}

Patient characteristics correspond to an average HS population. Hurley stages were evenly distributed (Table 1). Mean age at 
Table 1. Standardized questions.

\begin{tabular}{ll}
1 & When did you have your first accesses? \\
2 & When were you diagnosed of having HS? \\
\hline 3 & What is your current weight? Did it change during the time you have been suffering from HS? \\
4 & Do you smoke? If yes, since when? If no have you smoked before? Did you notice any change in HS severity when you were not smoking? \\
\hline 5 & Do you suffer from any form of digestive problem? (flatulence, pain, nausea, diarrhea, obstipation?) Have you tried any diet? \\
6 & Do you sweat a lot in your skin folds? \\
\hline 7 & Do you shave your skin folds? \\
\hline 9 & Do you use any sort of skin care for your skin folds? \\
\hline 10 & Does anyou identified any sort of trigger factor that aggravates HS in your view? \\
\hline HS, hidradenitis suppurativa.
\end{tabular}

onset of HS was 25.4 years. Interestingly, there was a significant difference $(\mathrm{P}=0.0064)$ between age at onset in familial cases (21.5 years) and sporadic cases (28.3 years) (Table 2).

Patients did not show very high body mass index (BMI) values. $30 \%$ of patients had BMI values $>30$ indicating obesity. Only $15 \%$ of patients showed severe obesity as indicated by a BMI $>35$. There was no correlation of disease severity as indicated by Hurley stage with BMI (obese vs nonobese). $35 \%$ complained of digestive problems and had tried different sorts of diet. We identified 2 cases of gluten-sensitive enteropathy, in which HS improved considerably after introduction of gluten free diet. In 7 further patients, introduction of low dairy/low carbohydrate diet considerably improved HS.

$77.5 \%$ had never used any sort of skin care in the intertriginous areas except disinfection or shower gels.

We found $67 \%$ of our patients to be current smoker and $82.5 \%$ ever-smoker which is well in line with previous studies. However, we also determined the time gap $(\Delta \mathrm{Nic} / \mathrm{HS})$ between onset of smoking and onset of HS. On average, patients had started smoking 8 years before the first abscesses appeared. In 3 patients, abscesses appeared before smoking was started. Only 2 patients had seen an immediate connection between smoking behavior and HS intensity. There was a significant difference $(\mathrm{P}=0.0345)$ of the $\Delta \mathrm{Nic} / \mathrm{HS}$ between familial $(\Delta \mathrm{Nic} / \mathrm{HS}=4.8$ years $)$ and sporadic forms ( $\Delta \mathrm{Nic} / \mathrm{HS}=10.6$ years $)$. Digestive problems were found in $40 \%$ of the patients. Of these, $7 / 15(46.7 \%)$ had tried different diets. $8 / 25$ (32\%) of patients who had not complained of digestive problems, also had tried various diets. Diets mentioned mostly consisted of omission of milk, lactose, sugar, nightshades, wheatmeal products or grains in general.

In 2 patients that had not complained of
Table 2. Patient characteristics.

\begin{tabular}{lc} 
Patient characteristics & $\mathbf{N}=\mathbf{4 0}$ \\
Male & 24 \\
Female & 16 \\
\hline Mean age & 38.9 years \\
Mean age at onset of HS & 25.4 years \\
$\quad$ Mean age at onset of HS familial HS & 21.5 years \\
$\quad$ Mean age at onset of HS sporadic HS & 28.3 years \\
\hline Mean duration of HS & 13.3 years \\
Positive family history of HS & $17(42.5 \%)$ \\
\hline Mean Hurley Stage & 2.1 \\
Hurley 1 & 10 \\
Hurley 2 & 15 \\
Hurley 3 & 14 \\
Mean BMI & 27.3 \\
BMI $<25$ & $14(35 \%)$ \\
BMI 25-30 & $12(30 \%)$ \\
BMI 30-35 & $6(15 \%)$ \\
BMI >35 & $6(15 \%)$ \\
\hline
\end{tabular}

$\mathrm{N}$, number; HS, hidradenitis suppurativa; BMI, body mass index.

Table 3. Trigger factors.

\begin{tabular}{lc}
\hline Trigger factors & $\mathrm{N}=40$ \\
Current smoker & $27(67.5 \%)$ \\
Ever smoker & $33(82.5 \%)$ \\
\hline Mean age at onset of smoking & 16.7 years \\
Mean age at onset of HS & 25.4 years \\
\hline$\Delta$ Nic/HS all patients & 8 years \\
$\Delta$ Nic/HS familial HS & 4.8 years \\
\hline$\Delta$ Nic/HS sporadic HS & 10.6 years \\
Digestive problems (flatulence, diarrhea, pain, nausea) & $15(40 \%)$ \\
\hline Digestive problems: previous diet (any) & $7 / 15(46.7 \%)$ \\
No digestive problems: previous diet (any) & $8 / 25(32 \%)$ \\
\hline Previous skin care of the flexures (any lo-tion, cream) & $9(22.5 \%)$ \\
Shaving of affected areas (shave or trim) & $23(57.5 \%)$ \\
\hline Sweating increased in skin folds & $23(57.5 \%)$ \\
Stress as potential trigger & $20(50 \%)$ \\
\hline
\end{tabular}

$\mathrm{N}$, number; HS, hidradenitis suppurativa; $\Delta \mathrm{Nic} / \mathrm{HS}$, time difference between onset of nicotine abuse and onset of $\mathrm{HS}$. 
digestive problems, celiac disease could be diagnosed. After introduction of a glutenfree diet HS lesions cleared largely. One patient with digestive problems suffered from Crohn's disease.

Skin care of the flexures with any kind of lotion or cream was only performed by a small minority of patients (22\%). Most had only used shower gel or disinfectants. More than half of the patients $(57.5 \%)$ shaved the affected areas or had done so for a long time. Only 3 considered shaving an aggravating factor for HS. $57 \%$ of patients complained of increased sweating in the flexures. There was no connection to HS flares or intensity. Stress was mentioned as trigger by half of the patients (Table 3 ).

More than $60 \%$ of patients considered early intervention with a fusidic acid/betamethasone cream (FA/B) a helpful measure, as described earlier. ${ }^{9}$ Patients had been instructed to apply FA/B twice daily on pustules, papules, small nodules, erythematous areas induced by irritation of any kind. 32.5\% had not used FA/B for various reasons. There was no difference Hurley stage between users and non-users. Only 2 patients did not find any benefit in using FA/B. Side effects of FA/B were not reported. In addition to FA/B treatment, weight loss (1 patient), diet (3 patients), reduction of irritation by change of clothing style (1 patient), improvement of skin care (2 patients) or laser epilation (4 patients) were reported as successful measures to reduce HS inflammatory intensity and frequency of new abscess formation. Altogether $70 \%$ of patients judged preventive measures that had been suggested to be beneficial. 10 $(25 \%)$ patients had not applied the suggested measures or were not able to judge their success for various reasons. Only 2 patients found any suggested preventive measure fruitless (Table 4).

\section{Discussion}

Many different research groups have addressed comorbidities and risk factors of HS. ${ }^{3,4,7,10,11}$ It is firmly established that obesity, smoking, stress, poor hygiene and mechanical stress are associated with HS. Other factors like sweating or the use of deodorants are less intensely studied. ${ }^{12}$ It is difficult to discern factors only associated with HS from factors causing or aggravating HS.

In a questionnaire based approach a British working group analyzed the natural history of HS and found worsening and alleviating factors. ${ }^{10}$ Heat, sweating and stress were the most commonly cited fac-

Table 4. Success of preventive measures.

\begin{tabular}{lc} 
Prevention & $\mathbf{N}=40$ \\
Application of FA/B helpful & $25(62.5 \%)$ \\
Any preventive measure successful & $28(70 \%)$ \\
\hline Success of preventive measure cannot be judged & $10(25 \%)$ \\
Preventive measure without effect & $2(5 \%)$ \\
\hline
\end{tabular}

$\mathrm{N}$, number; FA/B, fusidic acid/betamethasone combination cream.

tors to exacerbate the disease and $32 \%$ of patients noted a deterioration of HS in the summertime. In our study, $57 \%$ of patients had noticed and complained of an increased amount of sweating in the flexures. However, only 3 patients considered sweating as trigger factor for HS. Sweating may aggravate friction and irritation and sweat serves as a transporter of nicotine and other potentially irritating agents. ${ }^{3}$ Treatment of perspiration using deodorants has not been helpful in one study. ${ }^{11}$ However, in that study, it was not recorded, what kind of deodorants had been used and whether irritation had occurred as a consequence of their use. It is well known that aluminium salt-containing deodorants have an inherent irritative potential. ${ }^{13}$ Hence, deodorants are even listed as aggravating factors by $13 \%$ of patients. ${ }^{10}$ So far there are no reliable data regarding systemic antiperspirant therapies (e.g. using anticholinergics).

An association between shaving the affected areas and earlier disease onset has been shown. ${ }^{10,12}$ Shaving itself as well as the regrowing hairshafts frequently lead to irritation and folliculitis in mechanically stressed areas like the skin folds, thus initiating the inflammatory cascade of HS. ${ }^{14}$ Hence, shaving just like any other sort of friction or mechanical stress should be avoided. Since inflammation starts at the hair follicle, the simple logic that absence of terminal hair follicles prevents HS lesions from forming is compelling. Some clinical studies support this hypothesis. 22 patients with Hurley stage II to III disease were treated with a Neodym-YAG Laser device repeatedly. A $65 \%$ decrease in disease severity after 3 monthly treatments, was observed. Other hair reduction lasers have also been used with success in several small case series: an 800-nm diode laser, intense pulsed light therapy and a ruby laser. ${ }^{15}$ Taken together the results justify a recommendation of permanent laser assisted hair reduction in early stages of HS, thus fulfilling the criteria for prevention. In our study 4/40 patients had undergone laser assisted hair removal with good success.

Strengthening the epidermal barrier and thus influencing the innate immune system and antimicrobial peptide production is a basic thought and observation learned from the treatment of atopic dermatitis. ${ }^{16}$ By regular application of body lotions and creams known from the treatment of atopic dermatitis, the barrier of HS patients' skin folds may be stabilized. Surprisingly in our patient collective, only a minority of patients $(22.5 \%)$ had thought of that simple measure before. In 2 patients HS improved only with improvement of skin care. Generally, the role of topical skin care tends to be underestimated by both patients - like in our study - and treating (non-dermatologist) physicians.

There is international consensus that HS is not an infectious but an inflammatory disease..$^{2-4,7}$ This has been corroborated by the lack of serious infections in HS patients treated with anti-TNF therapies. ${ }^{1}$ However, there is conflicting data on the role of bacterial pathogens involved..$^{3,4}$ Based on the altered microbiome of HS there are several complex systemic antibiotic regimens to treat HS, to note, with some success. ${ }^{4}$ Likewise, antiseptics and topical antibiotics are used routinely in HS. However, the effect of using antiseptics with regard to microbiome composition and flare reduction has not been examined intensely and should be reconsidered. The topical antiseptic triclosan seems to be of some benefit in combination with oral high dose zinc medication. ${ }^{17}$ In a retrospective study, topical antibiotics had been used by $67 \%$ of patients. ${ }^{18}$ Topical administration of clindamycin reduces abscess-, nodule- and pustule formation and its use is recommended in the European HS guideline. ${ }^{2}$ Fusidic acid cream is less well established but has proven its beneficial effect on HS in a large prospective study. ${ }^{19}$

Infundibulofolliculitis is the primary HS lesion ${ }^{3}$ and its early treatment is mandatory for prevention of more severe nodules of abscesses. Since HS is not an infectious but an inflammatory disease, there is a potential role for a combination of topical steroids and antibiotics. There are different candidates on the market including a fix combination of fusidic acid and betamethasone valerate $0.1 \%(\mathrm{FA} / \mathrm{B})$, which exerts T- 
cell-specific immunosuppressive properties similar to those of cyclosporine. ${ }^{20}$ Another licensed candidate is a fix combination of gentamicinsulfate and betamethasondipropionate, which we have found useful in the management of HS. Combination creams exert stronger anti-inflammatory action than antibiotic creams alone and seem to be of considerably beneficial effect for HS, if treatment is initiated in time. This is corroborated by our patients' judgment on the effect of $\mathrm{FA} / \mathrm{B}$ cream treatment. $62.5 \%$ found the application of FA/B helpful in the management of HS. ${ }^{9}$ This figure probably underestimates the real power of $\mathrm{FA} / \mathrm{B}$ in HS since $25 \%$ of patients had not used FA/B or could not attribute an observed impact on HS only to FA/B usage. Clearly, controlled, prospective studies are needed to corroborate these observations.

There is a significant association of HS with obesity, with $60-77 \%$ of HS patients being overweight, and $20-30 \%$ obese. However, especially in familial HS cases, obesity does not seem to prevail..$^{3,4}$ In our patient group 30\% were overweight (BMI $>25<30)$ and $30 \%$ obese $($ BMI $>30)$. There was no correlation between body weight and family history (not shown). Obese patients have larger inverse skin areas leading to enhanced friction and mechanical stress. Obesity also facilitates sweat retention, which may aggravate mechanical stress. In addition, obesity is related to increased levels of pro-inflammatory cytokines in the blood (e.g. IL-1, IL-6, $\mathrm{TNF} \alpha$ ), whereby both IL-6 and TNF $\alpha$ are derived from adipocytes. ${ }^{3,4}$ Reduction of adipose tissue by weight loss through bariatric intervention improved HS severity dramatically. ${ }^{21}$ Altogether it seems safe and effective to suggest to obese HS patients weight loss as means of preventing disease progression. Frequently also non-obese patients are searching for something wrong in their diet which causes HS. Diet may be part of a weight loss program and as such beneficial. There are various diets that have been informally trialled and the results are being shared within the HS community. Unfortunately, only few controlled studies have been performed. In a small uncontrolled study, $39(=83 \%)$ of 47 patients improved under dairy-free diet and none worsened. $^{22}$ Dairy products and highly refined carbohydrates have been proposed to influence acne vulgaris by promoting anabolic and pro-inflammatory signaling cascades. ${ }^{23}$ A similar influence could play a role in HS. In our study, patients with digestive problems were more likely to test any form of diet. However, a considerable number of patients without digestive problems had also tested different sorts of diets, most- ly by omission of milk, lactose, sugar, night-shades, wheatmeal products or grains in general.

While we have no reliable data on the validity of the different proposed diets, it is worthwhile to carefully explore the patients' eating habits. On the basis of a possible role of intestinal hyperpermeability, ${ }^{24}$ HS patients may be advised to reduce potentially harmful diet components like wheat and alcohol. If available, a dietician may propose a low caloric, low dairy diet based on individual sensitivities. Especially obese patients should be encouraged to lose weight. To date, there are no data available on a possible connection between celiac disease and HS. However, in two patients of our collective (and one more HS patient outside this collective) we found previously unrecognized celiac disease that improved together with HS on a gluten free diet, underlining the importance of a healthy gut for inflammatory diseases. ${ }^{24}$ We suggest to routinely screen HS patients for celiac disease in future trials. Clearly further data is needed before a general recommendation for a specific HS diet can be given.

There is a significant association between smoking habits and HS. Up to $90 \%$ of HS patients are smokers or ex-smokers suggesting that tobacco is a potential trigger for HS. ${ }^{3,4}$ Nicotine is one of the major toxins of cigarette smoke and in vitro data suggest a potential role of the non-neuronal cholinergic system in promoting infundibular epithelial hyperplasia and thus follicular plugging. ${ }^{3}$ Nicotine may act on the nicotinic acetylcholine receptors ( $\mathrm{nAChR}$ ) present on the different immune cells present in HS lesions. Clinically, the severity of disease response to therapy and frequency of recurrence sometimes correlates with smoking habits. ${ }^{25,26}$ In one study, smokers have been shown to have higher Sartorius scores than former smokers than non-smokers. ${ }^{11}$

However, HS may start long before smoking and there is a number of nonsmoking HS patients. HS severity did not correlate with smoking status in a large French study. ${ }^{11}$ Few studies have correlated smoking habits with the time course of HS and disease severity. In one study, ${ }^{27} 32$ of 56 patients had started smoking before the outbreak of HS (on average 10.4 years before the first symptoms occurred). 10 patients became active smokers after the onset of the disease (on average 5.2 years after the first symptoms occurred). This is well in line with our data. We also found a time gap between onset of smoking and onset of HS $(\Delta \mathrm{Nic} / \mathrm{HS})$ of 8 years. Likewise, only a small minority of patients found alleviation of symptoms in direct association with smoking cessation (not shown). The large
$\Delta \mathrm{Nic} / \mathrm{HS}$ of $8-10$ years provokes new research into long term consequences of smoking that might be related to HS pathogenesis. Likewise, long term deleterious effects may require long term tissue repair, explaining the lack of correlation between smoking cessation and HS disease activity. So far, no study has correlated smoking status data with genetic data, i.e. familial or sporadic forms of HS. In our collective, there was a significant difference $(\mathrm{P}=0.0345)$ of the $\Delta \mathrm{Nic} / \mathrm{HS}$ between familial $(\Delta \mathrm{Nic} / \mathrm{HS}=4.8$ years $)$ and sporadic forms $(\Delta \mathrm{Nic} / \mathrm{HS}=10.6$ years) even though there was no significant difference as far as age at onset of smoking is concerned (not shown). This may indicate a higher vulnerability of familial HS towards an additional factor, contained in cigarette smoke. Smoking cessation should be encouraged for general reasons especially if a metabolic syndrome is present, since it is a well-known and strong risk factor for cardiovascular diseases. ${ }^{28}$ However, immediate beneficial or preventive effects on HS disease intensity should not be expected or promised. Long term studies on smoking in HS and a better understanding of HS pathomechanisms are clearly needed.

\section{Conclusions}

In conclusion, even though we do not understand all aspects of HS pathogenesis, we know influencing and associated factors. The knowledge of these different factors may help discriminate patients that profit from different treatment and preventive approaches. Stopping smoking, losing weight, eating healthy, reducing mechanical stress, strengthening the skin barrier by regular skin care and addressing psychosocial difficulties could make a difference.

HS certainly is a heterogenous multifactorial disease. Thus, careful patient analysis is warranted before preventive recommendations should be given. Identifying familial cases and the exact time line and relationship in between HS and the use of tobacco, prevents us from overestimating the role of smoking in the course of the disease. Smoking cessation may in an individual case not lead to improvement of HS. A normal weight HS patient does not have to be intensely informed on weight loss strategies. Rather, eating habits should be analyzed and possible aggravating and alleviating factors discussed with the patient. Telling the complex truth to the patient is mandatory if we are not to lose the patients' confidence, which is dearly needed should more severe disease stages come into focus. 
Secondary prevention means, we have to identify individual trigger factors together with the patient in order to prevent HS from progressing. Our study, for the first time, shows that secondary prevention of HS is possible already today and beneficial for the patient.

\section{References}

1. Kimball AB, Kerdel F, Adams D, et al. Adalimumab for the treatment of moderate to severe hidradenitis suppurativa: a parallel randomized trial. Ann Intern Med 2012; 157:846-55.

2. Zouboulis CC, Desai N, Emtestam L, et al. European S1 guideline for the treatment of hidradenitis suppurativa/acne inversa. J Eur Acad Dermatol Venereol 2015;29:619-44.

3. Kurzen H, Kurokawa I, Jemec GB, et al. What causes hidradenitis suppurativa? Exp Dermatol 2008;17:455-72.

4. Sabat R, Tsaousi A, Rossbacher J, et al. Acne inversa/hidradenitis suppurativa: an update. Hautarzt 2017;68:999-1006.

5. Kligman AM. Welcome letter. 2nd International Conference on the Sebaceous Gland, Acne, Rosacea and Related Disorders. Sep 13-16 2008, Rome, Italy.

6. Feinleib M. A dictionary of epidemiology. Fourth Edition - Edited by John M. Last, Robert A. Spasoff, and Susan S. Harris. Am J Epidem 2001;154:93-4.

7. Alikhan A, Sayed C, Alavi A, et al. North American clinical management guidelines for hidradenitis suppurativa: a publication from the United States and Canadian Hidradenitis Suppurativa Foundations. Part I: Diagnosis, evaluation, and the use of complementary and procedural management. J Am Acad Dermatol 2019;81:76-90.

8. Kurzen H. Secondary prevention of hidradentitis suppurativa/acne inversa. Exp Derm 2016;25:7.
9. Kurzen H. Usefulness of a fix combination cream of fusidic acid and betamethasone in the treatment of hidradenitis suppurativa Exp Dermatol 2017;26:24.

10. von der Werth JM, Williams HC. The natural history of hidradenitis suppurativa. J Eur Acad Dermatol Venereol 2000;14:389-92.

11. Canoui-Poitrine F, Revuz JE, Wolkenstein $\mathrm{P}$, et al. Clinical characteristics of a series of 302 French patients with hidradenitis suppurativa, with an analysis of factors associated with disease severity. J Am Acad Dermatol 2009;61:51-7.

12. Morgan WP, Leicester G. The role of depilation and deodorants in hidradenitis suppurativa. Arch Dermatol 1982;118:101-2.

13. Swaile DF, Elstun LT, Benzing KW. Clinical studies of sweat rate reduction by an over-the-counter soft-solid antiperspirant and comparison with a prescription antiperspirant product in male panelists. $\mathrm{Br} \mathrm{J}$ Dermatol 2012;166:22-6.

14. Boer J, Nazary M, Riis PT. The role of mechanical stress in hidradenitis suppurativa. Dermatol Clin 2016;34:37-43.

15. Hamzavi IH, Griffith JL, Riyaz F, et al. Laser and light-based treatment options for hidradenitis suppurativa. J Am Acad Dermatol 2015;73:78-81.

16. Furue M, Chiba T, Tsuji G, et al. Atopic dermatitis: immune deviation, barrier dysfunction, IgE autoreactivity and new therapies. Allergol Int 2017;66:398403.

17. Hessam S, Sand M, Meier NM, et al. Combination of oral zinc gluconate and topical triclosan: An anti-inflammatory treatment modality for initial hidradenitis suppurativa. J Dermatol Sci 2016;84:197-202.

18. Ingram JR, McPhee M. Management of hidradenitis suppurativa: a UK survey of current practice. $\mathrm{Br} \mathrm{J}$ Dermatol
2015;173:1070-2.

19. Shirah BH, Shirah HA. Effective modified conservative tissue preserving protocol to treat stage I axillary hidradenitis suppurativa: a prospective cohort study of 627 patients with five years follow-up. J Dermatol Treat 2017;28:458-63.

20. Virgili A, Borghi A, Minghetti S, Corazza M. Comparative study on topical immunomodulatory and antiinflammatory treatments for plasma cell vulvitis: long-term efficacy and safety. J Eur Acad Dermatol Venereol 2015;29:507-14

21. Thomas CL, Gordon KD, Mortimer PS. Rapid resolution of hidradenitis suppurativa after bariatric surgical intervention. Clin Exp Dermatol 2014;39:315-7.

22. Danby FW. Diet in the prevention of hidradenitis suppurativa (acne inversa). J Am Acad Dermatol 2015;73:S52-4.

23. Melnik BC. Linking diet to acne metabolomics, inflammation, and comedogenesis: an update. Clin Cosmet Investig Dermatol 2015;8:371-88.

24. Fasano A. Leaky gut and autoimmune diseases. Clin Rev Allergy Immunol 2012;42:71-8.

25. Denny G, Anadkat MJ. The effect of smoking and age on the response to first-line therapy of hidradenitis suppurativa: an institutional retrospective cohort study. J Am Acad Dermatol 2017;76:54-9.

26. Sartorius K, Emtestam L, Jemec GB, Lapins J. Objective scoring of hidradenitis suppurativa reflecting the role of tobacco smoking and obesity. $\mathrm{Br}$ J Dermatol 2009;161:831-9.

27. König A, Lehmann C, Rompel R, Happle R. Cigarette smoking as a triggering factor of hidradenitis suppurativa. Dermatology 1999;198:261-4.

28. Borden WB, Davidson MH. Updating the assessment of cardiac risk: beyond Framingham. Rev Cardiovasc Med 2009; 10:63-71. 